\title{
Finding The Missing Piece: Content-Based Video Comparison
}

\author{
Maia Zaharieva, Matthias Zeppelzauer, Dalibor Mitrović and Christian Breiteneder \\ Vienna University of Technology, Interactive Media Systems Group \\ Favoritenstr 9-11/188-2, A-1040 Vienna, Austria \\ \{zaharieva,zeppelzauer,mitrovic,breiteneder\}@ims.tuwien.ac.at
}

\begin{abstract}
The contribution of this paper consists of a framework for video comparison that allows for the analysis of different movie versions. Furthermore, a second contribution is an evaluation of state-of-the-art, local feature-based approaches for content-based video retrieval in a real world scenario. Eventually, the experimental results show the outstanding performance of a simple, edge-based descriptor within the presented framework.
\end{abstract}

Keywords-content-based video comparison; framework; local features; SIFT; MPEG-7; differential descriptors; evaluation;

\section{INTRODUCTION}

The goal of automatic video comparison is the identification of redundant material (sequences) in two videos and at the same time the identification of material that is unique. The compared videos may be two versions of the same feature film, e.g. the original cut and the director's cut or two different movies that share a particular amount of material, such as documentary films and compilation films. Applications of video comparison include the identification of sequences that have been removed (e.g. deleted scenes due to censorship or re-editing) and copy detection in order to reveal copyright violations.

One especially demanding area for video comparison is archive film material. Today, the original versions (filmstrips) of very old movies do often no longer exist. The remaining copies are usually backup copies from film archives that are often incomplete due to bad storage, mold, and film tears.

Automatic video comparison may assist in the reconstruction of the original version from the existing copies. Different versions of film archive material vary significantly not only by the actual content but also due to material-specific artifacts (dimension of film strip, dirt, liquids, low contrast, flicker, etc.). The movies are often monochromatic and do not contain sound which limits the set of available modalities and applicable techniques. Moreover, in practice, it is not always obvious if two shots are identical or not. Fig. 1 shows the challenge of identifying shot correspondences in film archives. Fig. 1(a) depicts the first frames of two identical shots that possess different appearance due to visible cue marks and scratches in the first shot, frame shifting in the second shot, and contrast differences in both shots. On the contrary, Fig. 1(b) shows the first frames of two similar and yet different shots with high perceptual similarity.

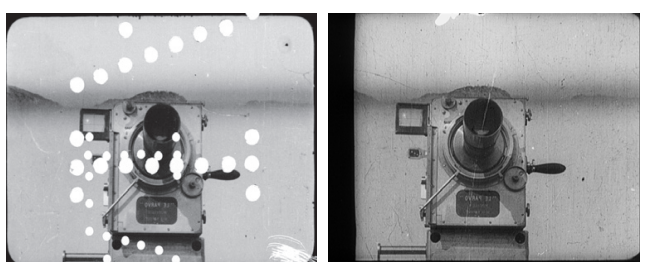

(a) Identical shots but different appearance

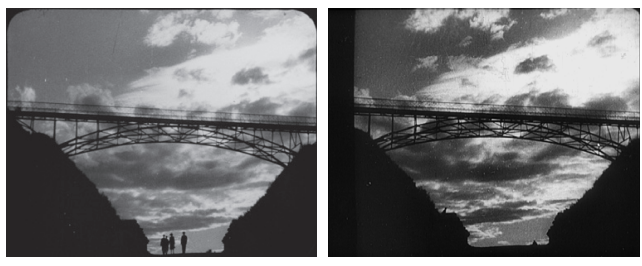

(b) Different shots despite high perceptual similarity

Figure 1. Identical vs. similar shots

Our contribution consists in a framework for video comparison that allows for the analysis of different movie versions. The framework does not need to be trained but operates fully automatically (without expert supervision). It exploits the hierarchical structure of video, i.e the frame, shot, and film level. The framework gives the user the flexibility to choose the appropriate hierarchy level for a given task. This makes the framework well-suited for different application scenarios such as the identification of missing shots or the reconstruction of the original film cut. Additionally, we employ several features for the description of video content and evaluate their robustness for low-quality archive films: the MPEG-7 Edge Histogram [1], Scale Invariant Feature Transform (SIFT) features [2], and differentialbased descriptors [3]. Experimental results show that the proposed framework yields high recognition rates and only few false positives for the investigated application scenarios. Furthermore, the knowledge about the hierarchical structure of a video allows for the outstanding performance of the simple, edge-based descriptor at much lower computational cost than state-of-the-art local feature-based descriptors.

This paper is organized as follows: Section II presents related work on video comparison. The proposed framework is described in Section III. Section IV outlines the methods used for the experiments presented in Section V. We conclude in Section VI and give an outlook for further research. 


\section{RELATED WORK}

By definition, a video copy is a transformed video sequence [4]. The transformation can be of technical nature (e.g. change in video format, frame rate, resizing, shifting, etc.) or editorial modification (frame insertion/deletion, background change, etc.). Existing approaches rely on the extraction of either global or local features which are matched against a reference video set. Lie et al. propose a compact binary signature based on color histogram for the recognition of TV commercials [5]. Zhang et al. use color moments and a stochastic attributed relational graph matching to detect duplicated news videos [6]. Both methods result in a compact feature representation and, thus, enable efficient search and indexing. However, they are not robust to illumination changes, cropping or partial occlusions. Local feature-based methods overcome these limitations and often achieve better performance. Sivic et al. use a combination of affine covariant regions and scale-invariant feature transform (SIFT) for object and scene matching and retrieval [7]. Laptev et al. propose spatio-temporal fingerprints for event detection [8]. Joly et al. apply the Harris corner detector and a differential description of the local region around each interest point [9]. This approach was shown to be superior over further methods used in the literature such as ordinal intensity signatures or space-time interest points [4]. Zhou et al. present a video similarity model which combines normalized chromaticity histogram and shot duration [10]. The proposed model requires color information and uses each frame of a shot to build its visual feature. A further limitation of the approach is that it is only robust to lowlevel transformations such as frame rate conversion and compression format. Sand and Teller propose an imageregistration method for aligning two videos recorded at different times into the spatio-temporal domain [11]. The authors combine interest point based matching and local motion estimation (KLT) for frame alignment. The proposed method has low invariance to affine transformation and high computational costs of several minutes per second of video.

Our work shows some similarities to the approach by $\mathrm{Ng}$ et al. [12]. The authors propose a tree matching algorithm based on the hierarchical structure of a video. Unlike our work, the authors define the video shot as the lowest hierarchical level of a video structure whereas each shot is represented by its first frame. Following, similarity is computed by a combination of color histogram and shot style (camera motion and the length of a shot). A significant limitation of this work is that it is only useful for comparison of videos which exhibit high distinctive pattern of motions and have not undergone strong modification (e.g. illumination changes or frame deletion/insertion).

Recent evaluations bear mainly two limitations. First, the used data sets comprise video clips of high quality and pre-defined fixed length between 5 and 60 sec. Sec- ond, the experiments are usually performed using synthetic transformations such as resizing, frame shifting, contrast and gamma modification, gaussian noise additions, etc. In contrast, the evaluation presented in this paper goes beyond the boundaries of a single video shot and accounts for the overall video coherence. The additional knowledge about the video structure allows for the definition of a straightforward and reliable framework for video comparison. Within this framework we compare state-of-the-art video copy detection algorithms with the MPEG-7 Edge Histogram [1]. Furthermore, our evaluation is based on real world scenarios on challenging video data exposing three different types of artifacts: 1) artifacts originating from the analog filmstrips (e.g. contrast and exposure changes, blurring, frame shift, dirt, film tears, etc.); 2) transformations (e.g. changes in video format, resizing, cropping, etc.) and 3) editorial operations such as frame/shot insertion and frame/shot deletion.

\section{FRAMEWORK}

Videos can be represented by a hierarchy of five levels: video, scene, group, shot and frame [13]. Video shot is a continuous sequence of frames recorded from a single camera delimited by shot boundaries. Video scene is a collection of semantically related and temporally adjacent shots. Video groups represent the intermediate level between physical shots and semantic scenes. We propose a framework which exploits the physical video structure, i.e. video-, shot- and frame-level. Fig. 2 visualizes the architecture and information propagation within the framework.

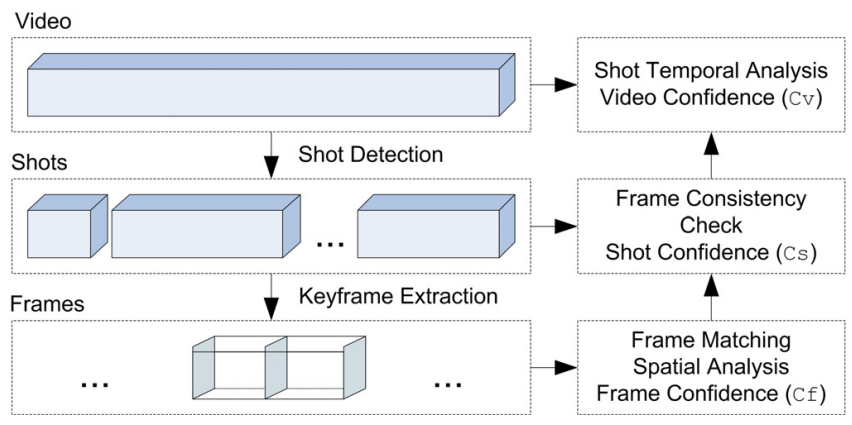

Figure 2. Framework architecture and information propagation.

\section{A. Frame-level}

Video frames form the basic physical building block of a video. Since a single frame represents a still image, manifold global and local image features can be applied to describe its content. For performance reasons, features are usually not extracted for each frame of the shot but only for keyframes. There are different approaches to select the keyframes of a shot. Simple approaches extract a single frame [12], the first and the last frames [13], or every $n$-th frame [14] as a 
keyframe for the shot. More sophisticated methods account for e.g. the global intensity of motion [15].

We select the first, middle and last frame as keyframes to achieve fast processing. Due to various editorial operations, corresponding shots in different video versions often have different length and extracted keyframes are not identical. However, the keyframes capture nearly the same context which should provide a sufficient basis for matching based on high discriminative and reliable features.

The similarity between frame features is used to assign a video frame to a shot (frame voting), i.e. each keyframe of the first video is compared to each keyframe of the second video. Dependent on the selected feature, various distance metrics can be used to measure the visual similarity. In our work, we apply nearest neighbor ratio matching based on Euclidean distances. Additionally, we compute frame confidence measure, $c_{f}$, based on the distance spreading of all matches, i.e. if all distances lie closely together, the corresponding match is considered less reliable. In contrast, an outlier suggests a high matching confidence:

$$
c_{f}=1-\frac{\bar{d}_{m}}{\bar{d}}
$$

where $\bar{d}_{m}$ is the mean matching distance of the matched features, and $\bar{d}$ the mean matching distance of all descriptors. Finally, the comparison for each keyframe results in a paired value $\left(c_{f}\right.$, vote $\left._{\text {shot }}\right)$.

\section{B. Shot-level}

Since a video shot consists of frames, three factors may influence the shot matching decision: frames' votes, corresponding frame confidences and, optionally, the temporal ordering of the frames. In our evaluation, at least two out of the three keyframes have to vote for the same shot, otherwise the shot is rejected and classified as unknown. The shot confidence, $c_{s}$, accounts for the confidence of the voting frames and is defined as the average of their scores:

$$
c_{s}=\frac{\sum_{i=1}^{n} s_{i} \times c_{f i}}{\sum_{i=1}^{n} s_{i}} \begin{cases}s=1 \\ s=0 & \text { for a voting frame }\end{cases}
$$

where $c_{f i}$ is the frame confidence of the $i$-th frame and $n$ the number of keyframes in the shot.

\section{Video-level}

The video-level represents the highest layer in the framework. Given the domain of video comparison, the corresponding shots in different videos build a well-defined sequence. This additional knowledge is used to eliminate matched shots, which do not fit in the overall ordering sequence. To detect outliers we apply local minima and maxima suppression on the sequence formed by the respective shot ids. Finally, the average confidence score of matched shots is defined as video confidence $c_{v}$.

\section{Methods COMPARED}

\section{A. MPEG-7 Edge Histogram}

The MPEG-7 Edge Histogram captures the distribution of orientations of the edges. It has proven to be effective for image similarity retrieval [16]. We divide the image into 16 non-overlapping sub-images. For each sub-image we create a local edge histogram with 5 bins. We distinguish between vertical, horizontal, 45 degree, 135 degree, and nondirectional edges. The edge histogram for the entire frame contains $16 \times 5=80$ bins [1].

The edge histogram is highly robust against frame displacements since it captures global information within each block. Additionally, the feature is invariant to flicker. On the other hand it captures high-frequency information which makes it prone to artifacts like scratches and dirt. The influence of these artifacts is usually low compared to the influence of the dominant and meaningful edges. However, global artifacts, such as tears across the entire frame are reflected in the feature.

\section{B. SIFT features}

Lowe introduced the SIFT features as a method for extracting local image descriptors that are highly discriminative for object recognition [2]. SIFT features are invariant to changes in image translation, scaling, and rotation and partially invariant to changes in the illumination and affine distortions. Furthermore, they outperform other descriptors such as steerable filters, differential invariants, complex filters, moment invariants, and cross-correlations [17].

SIFT features are based on gradient distribution in salient regions. Interest points are identified at peaks (local minima and maxima) of a Gaussian function applied in scale space. All keypoints with low contrast or keypoints that are localized at edges are eliminated using a Laplacian function. At each feature location, an orientation is selected by determining the peak of the histogram of local image gradient orientations. Subpixel image location, scale and orientation are associated with each SIFT feature vector. Finally, the resulting SIFT feature descriptor has 128 dimensions $(4 \times 4$ location grid $\times 8$ gradient orientations).

\section{Differential descriptors}

Recently, several authors report the outstanding performance of differential-based descriptors in the context of video copy detection [3], [4], [15]. In this evaluation we follow the work of Law-To et al. [3]. In a comparative study presented in [4] this approach was reported to outperform the algorithm proposed by Joly et al. [15]. 
Table I

EXPERIMENTAL VIDEO DATA

\begin{tabular}{|l|l|r|r|r|r|}
\hline Case study & Video & Length & Shots & Frames & Shared shots \\
\hline CS1 & V1 & $1: 26: 48$ & 1782 & 93.743 & 1741 \\
& V2 & $1: 28: 40$ & 1782 & 95.768 & \\
\hline CS2 & V1 & $1: 04: 44$ & 603 & 97.116 & 600 \\
& V2 & $1: 04: 45$ & 612 & 97.134 & \\
\hline
\end{tabular}

First, the Harris corner detector [18] is used to extract interest points on every frame. Second, local features are computed at four spatial positions around interest points as Gaussian differential decomposition of the graylevel signal until the second order:

$$
f=\left(\frac{\partial I}{\partial x}, \frac{\partial I}{\partial y}, \frac{\partial I}{\partial x \partial y}, \frac{\partial^{2} I}{\partial x^{2}}, \frac{\partial^{2} I}{\partial^{2} y}\right)
$$

The resulting feature is a 20 -dimensional descriptor. Finally, interest points along frames are associated to build trajectories which are described by the average descriptors. Unlike the original work described in [3], we do not associate each trajectory with a behavior label ("motion"/'background"). Due to the low video quality, bouncy and unsteady video sequences often exhibit high motion characteristics which may lead to mislabeling and, thus, misclassification.

\section{EXPERIMENTS}

\section{A. Video data}

Subject of the experiments are films by the Soviet AvantGarde film maker Dziga Vertov. Vertov's films are historical artistic documentaries from the 1920s and 1930s. The films have a distinctive structure characterized by a high number of short, repeating shots with high visual similarity.

We conduct two case studies. We first investigate two versions of a film with two different tape-based analog sources. Concretly, we employ a Dutch and an Austrian copy of the film Man with the Movie Camera (CS1). The two copies were derived from the original source with several decades in between. They differ greatly in the image quality and the censored content. The second case study focuses on the film Enthusiasm (CS2). The two versions originate from the same analog copy. One copy is the result of an effort to manually reconstruct the original film by adding and removing shots.

The ground truth for corresponding video shots in different video streams is provided by film experts and available a priori. The length of the shots is strongly varying from 1 to 1473 frames/shot. Table I summarizes further technical details of the video data.

Technically, we utilize frame-by-frame scans of the original $35 \mathrm{~mm}$ black-and-white filmstrips. The low quality of the material results from the fact that the filmstrips are multiplegeneration copies that were never intended to be used for other purposes than backups. Additionally, the state of the material has degraded significantly, during storage, copying, and playback over the last decades. Fig. 3 shows common artifacts often found in archive film material.
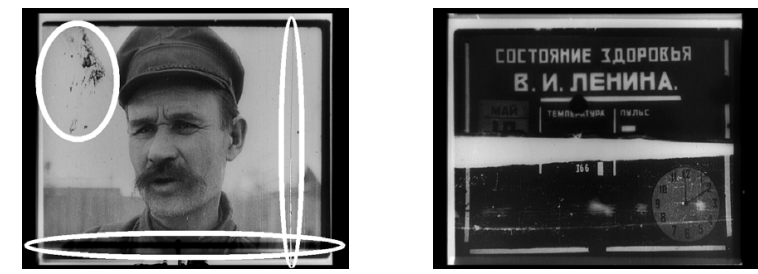

(a) Left: dirt (top left), vertical scratch (right), frameline copied into the image (bottom). Right: tear in the middle of the frame.
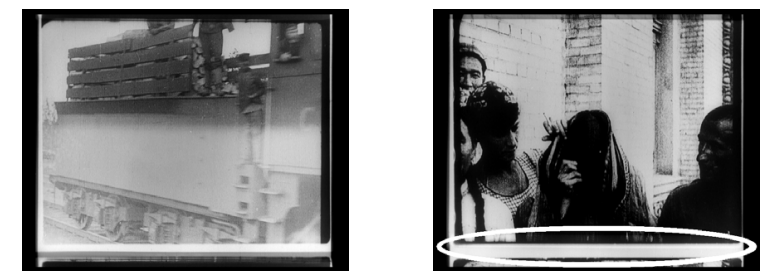

(b) Left: artifact introduced during copying (error in brightness). Right: unwanted high contrast, visible frameline (bottom).

Figure 3. Artifacts in archive film material.

\section{B. Experimental results}

For the evaluation of MPEG-7 Edge Histogram and SIFT features, we first extract 3 keyframes for each shot: the first, the middle, and the last one. Next, for each frame, feature descriptors are extracted according to the descriptions in Section IV-A and Section IV-B. The third approach, differential descriptors (DD), is based on feature trajectories and, thus, processes each frame of the shot (see Section IV-C for details on the implementation).

Local feature descriptors are matched by identifying the first two nearest neighbors in terms of Euclidean distances. A descriptor is accepted if the nearest neighbor distance ratio is below a predefined threshold. The misleading high number of matching descriptors penalizes algorithms which rely on the total number of matches. Thus, a further processing step is required to increase the reliability of detected matches. To overcome this problem, we ignore all ambiguous matches, i.e. those descriptors are eliminated that match several features in the other frame. Additionally, the RANdom SAmple Consensus (RANSAC) [19] algorithm is applied to remove outliers that do not fit a homography transformation. Finally, a video frame votes for the shot of the frame with the most matches. However, if less than $5 \%$ of the descriptors are matched or if the frame confidence is below $60 \%$, the match is considered unreliable and will be ignored, i.e. the frame is classified as unknown.

The evaluation of the results at frame-level for the MPEG7 Edge Histogram and the SIFT descriptor shows the supe- 
rior performance of SIFT features: averaged recall for the whole set of video data achieved $95 \%$ and the precision 94\% (see Table II). Surprisingly, MPEG-7 Edge Histogram proves to be a very competitive descriptor. In terms of average recall it even outperforms SIFT and scores $99 \%$. However, the average precision achieved $89 \%$ which is due to the higher number of false positives.

Table II

RECALL(R)/PRECISION(P) PERFORMANCE AT FRAME-LEVEL

\begin{tabular}{|c|c|c|c|c|}
\hline CS & \multicolumn{2}{|c|}{ MPEG-7 EH } & \multicolumn{2}{c|}{ SIFT } \\
& $\mathrm{R}$ & $\mathrm{P}$ & $\mathrm{R}$ & $\mathrm{P}$ \\
\hline CS1 & $99 \%$ & $84 \%$ & $94 \%$ & $92 \%$ \\
CS2 & $99 \%$ & $95 \%$ & $97 \%$ & $96 \%$ \\
\hline Aver. & $99 \%$ & $89 \%$ & $\mathbf{9 5 \%}$ & $\mathbf{9 4 \%}$ \\
\hline
\end{tabular}

At shot-level, confidence score of at least $70 \%$ achieved the best trade-off between recall and precision. Table III summarizes the results for all three approaches. Again, SIFT features dominate over the MPEG-7 Edge Histograms and the differential descriptors. Although the differential descriptors build on information from each frame of a given video shot, they show very low performance. This result is due to the nature of the experimental video data which requires exceptionally discriminative and reliable features. An analysis of the extracted differential descriptors shows low variance captured which results in their low distinctiveness. Hence, such descriptors are only feasible for highly discriminative data. In a scenario of multiple low quality video shots which belong to the same scene and show high visual similarity, this approach fails to correctly assign corresponding shots.

Table III

RECALL(R)/PRECISION(P) PERFORMANCE AT SHOT-LEVEL

\begin{tabular}{|c|c|c|c|c|c|c|}
\hline CS & \multicolumn{2}{|c|}{ MPEG-7 EH } & \multicolumn{2}{|c|}{ SIFT } & \multicolumn{2}{c|}{ DD } \\
& $\mathrm{R}$ & $\mathrm{P}$ & $\mathrm{R}$ & $\mathrm{P}$ & $\mathrm{R}$ & $\mathrm{P}$ \\
\hline CS1 & $93 \%$ & $93 \%$ & $95 \%$ & $95 \%$ & $48 \%$ & $47 \%$ \\
CS2 & $98 \%$ & $97 \%$ & $98 \%$ & $98 \%$ & $66 \%$ & $71 \%$ \\
\hline Aver. & $95 \%$ & $94 \%$ & $\mathbf{9 6 \%}$ & $\mathbf{9 6 \%}$ & $55 \%$ & $53 \%$ \\
\hline
\end{tabular}

Fig. 4 shows the plots of recall and precision versus the shot confidence measure $c_{s}$ for MPEG-7 Edge Histograms and SIFT features. Noteworthy is the stability of the SIFT descriptors. Recall begins to fall at confidence level of $40 \%$ whereas the critical value for the MPEG-7 Edge Histogram lies at about $65 \%$ (see Fig. 4(a)). Similarly, SIFT's precision decreases insignificantly while MPEG-7 Edge Histogram's precision rate drops about $4 \%$ (see Fig. 4(b)).

At video-level we account for the temporal alignment of matched shots and discard shots that do not fit in the ordering sequence. To detect outliers we apply peak detection (local minima and maxima) on the ordering sequence. Due to the lower number of false positives we expect a further increase of precision. Falsely detected peaks lead to a slight decrease in recall. This method completely fails when applied with the differential descriptors approach. The low performance of the approach does not allow for a clear discrimination between correct and incorrect peaks. Table IV summarizes the results of the performed video comparison for all three approaches.

Table IV

ReCALL(R)/PRECISION(P) PERFORMANCE AT VIDEO-LEVEL

\begin{tabular}{|c|c|c|c|c|c|c|}
\hline CS & \multicolumn{2}{|c|}{ MPEG-7 EH } & \multicolumn{2}{c|}{ SIFT } & \multicolumn{2}{c|}{ DD } \\
& $\mathrm{R}$ & $\mathrm{P}$ & $\mathrm{R}$ & $\mathrm{P}$ & $\mathrm{R}$ & $\mathrm{P}$ \\
\hline CS1 & $91 \%$ & $94 \%$ & $92 \%$ & $98 \%$ & $44 \%$ & $39 \%$ \\
CS2 & $97 \%$ & $98 \%$ & $97 \%$ & $99 \%$ & $59 \%$ & $68 \%$ \\
\hline Aver. & $93 \%$ & $96 \%$ & $\mathbf{9 3 \%}$ & $\mathbf{9 8} \%$ & $52 \%$ & $46 \%$ \\
\hline
\end{tabular}

Our last experiment investigates the identification of video shots that are unique. The SIFT descriptor correctly identifies $83 \%$ of all unique shots followed by the MPEG-7 Edge Histogram with $67 \%$ and the differential-based descriptors with $29 \%$. The large differences on a percentage basis are due to the low number of unique shots in our video data. Out of 44 unique shots only 24 are longer than 3 frames which is a basic requirement in our framework. Thus, the absolute difference of 4 shots between the performance of the MPEG-7 Edge Histogram and the SIFT descriptors is relatively low (see Table $\mathrm{V}$ for details).

Table V

UNIQUE SHOT DETECTION

\begin{tabular}{|l|r|r|r|r|}
\hline & Unique shots & MPEG-7 & SIFT & DD \\
\hline CS1 & 2 & 2 & 2 & 1 \\
CS2 & 22 & 14 & 18 & 6 \\
\hline Aver. & & $67 \%$ & $\mathbf{8 3 \%}$ & $29 \%$ \\
\hline
\end{tabular}

The results of the performed experiments put the competition between global and local descriptors into perspective. SIFT features are very discriminative and reliable and thus the amount of data to be explored can be reduced significantly. Despite the low quality and partially large differences between corresponding shots, just three frames per shot are sufficient to correctly assign them. However, MPEG7 Edge Histogram is more competitive than expected. The achieved performance is only marginally lower than SIFT while computational costs are significantly lower.

\section{CONCLUSION}

In this paper we presented a framework for video comparison which accounts for the overall video coherence. Within this framework we compared state-of-the-art video copy detection algorithms with MPEG-7 Edge Histogram. We presented the results of an evaluation based on a real world scenario on a challenging data set of archive film material. Despite the low video quality and various technical and editorial modifications of the data, MPEG7 Edge Histogram showed high recognition performance with accuracy comparable to top-performing local featurebased descriptors, but at much lower computational costs. 


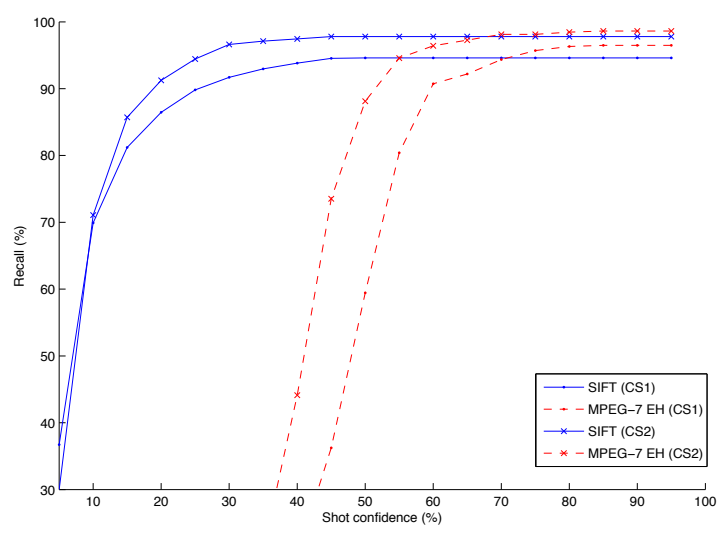

(a) Recall vs. shot confidence

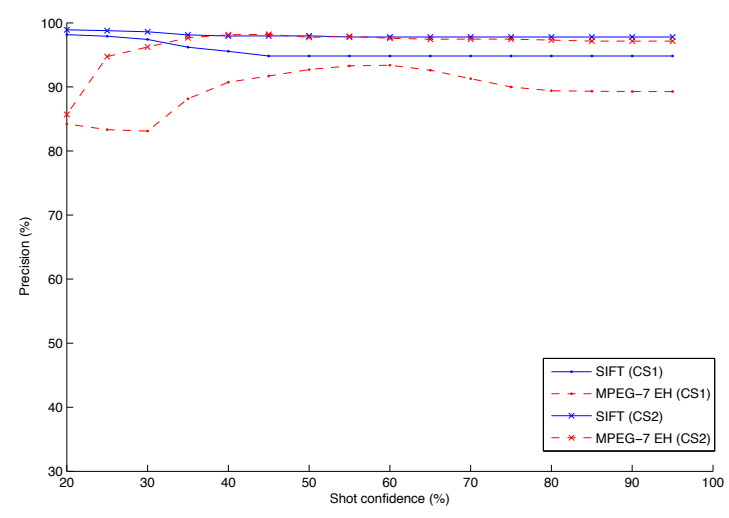

(b) Precision vs. shot confidence

Figure 4. Recall/precision vs. shot confidence at shot-level.

Moreover, the edge histogram significantly outperformed the state-of-the-art approach based on differential descriptors and is only marginally lower than SIFT.

\section{ACKNOWLEDGMENT}

This work was partly supported by the Vienna Science and Technology Fund (WWTF) under grant no. CI06 024.

\section{REFERENCES}

[1] ISO-IEC, Information Technology - Multimedia Content Description Interface - part 3: Visual. ISO/IEC, Moving Pictures Expert Group, 2002, no. 15938-3.

[2] D. G. Lowe, "Distinctive image features from scale-invariant keypoints," IJCV, vol. 60, no. 2, pp. 91-110, 2004.

[3] J. Law-To, O. Buisson, V. Gouet-Brunet, and N. Boujemaa, "Robust voting algorithm based on labels of behavior for video copy detection," in ACM Int. Conf. on Multimedia, 2006, pp. 835-844.

[4] J. Law-To, L. Chen, A. Joly, I. Laptev, O. Buisson, V. GouetBrunet, N. Boujemaa, and F. Stentiford, "Video copy detection: a comparative study," in ACM Int. Conf. on Image and Video Retrieval, 2007, pp. 371-378.

[5] Y. Li, J. Jin, and X. Zhou, "Video matching using binary signature," in Int. Symp. on Intelligent Signal Processing and Communication Systems, 2005, pp. 317-320.

[6] D.-Q. Zhang and S.-F. Chang, "Detecting image nearduplicate by stochastic attributed relational graph matching with learning," in ACM Int. Conf. on Multimedia, 2004, pp. 877-884.

[7] J. Sivic and A. Zisserman, "Video google: a text retrieval approach to object matching in videos," in IEEE Int. Conf. on Computer Vision, vol. 2, 2003, pp. 1470-1477.

[8] I. Laptev and T. Lindeberg, "Space-time interest points," in IEEE Int. Conf. on Computer Vision, 2003, pp. 432-439.
[9] A. Joly, C. Frélicot, and O. Buisson, "Robust content-based video copy identification in a large reference database," in Int. Conf. on Image and Video Retrieval, vol. 2728/2003. LNCS, 2003, pp. 414-424.

[10] J. Zhou and X.-P. Zhang, "Automatic indentification of digital video based on shot-level sequence amtching," in ACM Int. Conf. on Multimedia, 2005, pp. 515-518.

[11] P. Sand and S. Teller, "Video matching," ACM Trans. on Graphics, pp. 592-599, 2004.

[12] C. W. Ng, I. King, and M. R. Lyu, "Video comparison using tree matching algorithm," in Int. Conf. on Imaging Science, Systems and Technology, 2001, pp. 184-190.

[13] Y. Rui, T. S. Huang, and S. Mehrotra, "Constructing tableof-content for videos," Multimedia Systems, vol. 7, no. 5, pp. 359-368, 1999.

[14] O. Chum, J. Philbin, M. Isard, and A. Zisserman, "Scalable near identical image and shot detection," in ACM Int. Conf. on Image and Video Retrieval, 2007, pp. 549-556.

[15] A. Joly, C. Frélicot, and O. Buisson, "Content-based copy detection using distortion-based probabilistic similarity search," IEEE Trans. on Multimedia, vol. 9, no. 2, pp. 293-306, 2007.

[16] B. S. Manjunath, J. rainer Ohm, V. V. Vasudevan, and A. Yamada, "Color and texture descriptors," IEEE Trans. on Circuits and Systems for Video Technology, vol. 11, pp. 703$715,2001$.

[17] K. Mikolajczyk and C. Schmid, "A performance evaluation of local descriptors," IEEE PAMI, vol. 27, no. 10, pp. 16151630, 2005.

[18] C. Harris and M. Stephens, "A combined corner and edge detector," in Alvey Conference, 1988, pp. 147-152.

[19] M. A. Fischler and R. C. Bolles, "Random sample consensus: a paradigm for model fitting with applications to image analysis and automated cartography," Communications of the $A C M$, vol. 24, no. 6, pp. 381-395, 1981. 